\title{
Calculation of hydraulic resistance in the separator of the direct- flow control valve with a rotary lock
}

\author{
Anna Kapranova ${ }^{1, *}$, Anton Lebedev ${ }^{1}$, and Alexander Melzer ${ }^{2}$ \\ ${ }^{1}$ Yaroslavl State Technical University, Yaroslavl, Russian Federation \\ ${ }^{2}$ JSC «Regulyator», Yaroslavl, Russian Federation
}

\begin{abstract}
The purpose of this work is to analyze the coefficient of hydraulic resistance in the separator of a direct-flow control valve with a rotary lock according to the approximation of the superposition of pressure losses in elementary local resistances. In contrast to the known methods of constructing simulation models, the proposed analytical method of calculation is based on a qualitative assessment of the specified coefficient in the implementation of throttling of fluid flows in the "separator-rotary lock" unit, depending on the design and operating parameters of the process. It was found that, within the selected range of variation of the separator parameters, an increase in the valve opening degree from $20 \%$ to $50 \%$ leads to a decrease in the hydraulic resistance coefficient by 19.6 times, and an increase in this degree from $20 \%$ to $100 \%$ is associated with a $42.4-$ fold decrease in the studied characteristic. This circumstance justifies the effectiveness of the proposed method for throttling the flows of the working medium. The results obtained are used to study the influence of the main design parameters of the "separator-butterfly valve" unit on the flow capacity of the control valve and are relevant for stochastic modeling of the hydrodynamic cavitation process.
\end{abstract}

\section{Introduction}

The process of regulating fluid flows during the operation of pipeline valves requires the fulfillment of a number of regulatory conditions, which, first of all, include various conditions for selecting the flow rate characteristics and throughput of valves [1,2]. The presence of cavitation [3-5] during the operation of regulating devices additionally complicates this choice and leads to the need to make constructive decisions aimed at preventing the negative consequences of this phenomenon [1,2]. The presence of cavitation [3-5] during the operation of regulating devices additionally complicates this choice and leads to the need to make constructive decisions aimed at preventing the negative consequences of this phenomenon [1,2]. In particular, devices for throttling fluid flows using single [6,7] or stepped [8] perforated elements are widely used, for example, in direct-flow valves [6-9], including those with various shapes of throttling channels.

The actual problem of ensuring a given profile of the flow characteristic of a control valve when changing the degree of its opening [10] at the design stage of control equipment is directly related to the calculation of the hydraulic resistance coefficient for fluid flows in the flow part [11-13]. In addition, the methods for calculating the rational ranges of changes in the values of the parameters of the throttling process of liquid flows are also based on data on the coefficient of hydraulic resistance in the main unit of the control valve [14,15].
The purpose of this work is to analyze the coefficient of hydraulic resistance in the separator of a direct-flow control valve with a rotary lock [16] according to the approximation of the superposition of pressure losses in elementary local resistances.

The study of the dependence of hydraulic resistance in laminar and turbulent flow regimes of working fluid media on various factors is devoted to many works [13, 17-21]. However, the theory of turbulent flows belongs to a developing scientific direction, which contributes to the development of several types of methods for calculating the hydraulic resistance coefficient: theoretical and experimental [11-13,17], simulation [1821], analytical [13].

The first group of methods [11-13,17] is characterized by active involvement of experimental data in calculations, including in the form of empirical dependences of a tabular and graphical nature. In particular, in [17], an empirical formula was proposed for calculating the value of the hydraulic resistance coefficient in smooth channels taking into account a set of criteria (Reynolds, Froude and the degree of flow propagation)

The second group of methods [18-21] uses both ready-made software products and various difference schemes to obtain a numerical solution of the system of classical equations of continuity, motion and energy conservation for an incompressible working medium with special properties in channels of various shapes. For example, in [18], the case of the dependence of the local 
hydraulic resistance in a flat channel with a sudden narrowing on the temperature parameter was numerically investigated. Within the framework of numerical modeling in [19], the influence of wall roughness and rotational effects on the formation of turbulent flows in the channel was studied.

The third method [13], like the first group of methods [11-13,17], involves the implementation of the principle of superposition of pressure losses in elementary local resistances. This takes into account the features of the flow of the working fluid in the flow channels of specific devices of a simplified form, depending on the design parameters [13]. The advantage of analytical methods is the clarity of the results and the possibility of their application, for example, in stochastic modeling of hydrodynamic cavitation [22-24] in the working part of the control valve. In particular, for a direct-flow device with a movable external gate [25], the description of the integral characteristics [26] of the process under study is carried out taking into account the results of calculations [13] for the indicated coefficient.

In contrast to the known methods of constructing simulation models [18-21], the proposed analytical method of calculation is based on a qualitative assessment of the specified coefficient in the implementation of throttling of fluid flows in the "separator-rotary lock" [16] unit, depending on the design and operating parameters of the process. At the same time, the analysis of the simulation results obtained by the authors [13, 22-24] for the case of throttling of fluid flows in the separator of an axial valve [25] allows us to put forward a working hypothesis about the most significant factors affecting the process under study in the main valve assembly [16]. These circumstances are decisive in the design of pipeline valves [27-30].

\section{Description of a set of design parameters of the main unit of a direct- flow valve with an external rotary valve}

The main unit of the direct-flow valve [16] consists of two coaxial perforated cylindrical shells: a fixed inner (separator) and a movable outer (gate) with the possibility of rotating the latter relative to the longitudinal central axis of symmetry. The degree of opening of the separator changes with the rotation of the shutter due to the overlapping of the round holes on both shells with the formation of throttling holes in the form of a double segment or circle. We give the following designations for the structural parameters of the elements. Let the set of the specified parameters of the separator include: the diameters of the separator: external $D_{\text {ext } 1}$ and internal $D_{\text {int } 1}$; the length of the perforated part $L_{1}=L$; diameter of radial throttling holes $d_{01}=d_{0}$; distances: between rows of these holes $\lambda_{11}=\lambda_{1}$ and between holes in any row $\lambda_{21}=\lambda_{2}$; wall thickness $\delta_{1}$; the number of throttling holes in one row $q_{1}$; the number of these rows $q_{2}$. The set of design parameters of the external butterfly valve includes: wall thickness $\delta_{2}$; the length of the perforated part $L_{2}=L$; diameter of radial throttling holes $d_{02}=d_{0}$; distances: between rows of these holes $\lambda_{12}=\lambda_{1}$ and between holes in any row $\lambda_{22}=\lambda_{2}$; bevel angle $\varphi$. The parameters of the cylindrical parts of the outer and inner valve bodies include: their outer diameters $D_{\text {ext } 01}$ and $D_{\text {ext } 02}$, accordingly, the wall thickness $\delta_{01}=\delta_{02}=\delta_{0}$.

Then the independent parameters of the main unit of the direct-flow valve are the following components of the set $U_{1}=\left\{U_{1 v}\right\}, v=\overline{1, n_{U_{1}}}$ :

$$
U_{1}=\left\{D_{\text {int } 1}, D_{\text {ext } 01}, D_{e x t 02}, L, d_{0}, \lambda_{1}, \lambda_{2}, \delta_{1}, \delta_{2}, q_{1}, q_{2}, \varphi\right\}
$$

Taking into account (1), the dependence of the nominal sectional area of the separator $\omega_{y}=\omega_{y}(\eta)$ and the nominal bore diameter $D_{y}=D_{y}(\eta)$ on the valve opening degree $\eta \in[0 ; 1]$ are determined by the expressions

$$
\omega_{y}(\eta)=\pi d_{0}^{2} q_{1} q_{2} \eta / 4 ; D_{y}(\eta)=\left[4 \omega_{y}(\eta) / \pi\right]^{1 / 2}
$$

In this case, the angle of rotation $\beta_{\eta}=\beta_{\eta}(\eta)$ for the outer perforated shell (gate) is related to the value $\eta$ by the following relationship

$$
\beta_{\eta}(\eta)=2 H\left[\psi_{\eta}(\eta)\right] / D_{i n t 1}
$$

Here, the value of the segment center angle $\psi_{\eta}(\eta)$ for the open part of the throttling hole with the radius $d_{0}$ corresponds to the solution of the equation $\pi \eta=\psi_{\eta}-\sin \psi_{\eta}$. The following designations are adopted:

$$
\begin{gathered}
W\left[\psi_{\eta}(\eta)\right] \equiv d_{0} \sin \left[\psi_{\eta}(\eta) / 2\right] \\
H\left[\psi_{\eta}(\eta)\right] \equiv W\left[\psi_{\eta}(\eta)\right] \operatorname{tg}\left[\psi_{\eta}(\eta) / 4\right] / 2
\end{gathered}
$$

\section{Modes and methods}

The calculation of pressure $\Delta P$ losses in the unit "separator - external rotary lock" for a direct-flow valve [16] can be performed by taking the approximation of the validity of the superposition principle for pressure $\Delta P_{v}$ losses in elementary local resistances [10-12]

$$
\Delta P=\sum_{v=1}^{s} \Delta P_{v}
$$

based on the Weisbach formula [10]

$$
\Delta P=\left(\rho_{L} / 2\right) \sum_{v=1}^{s} w_{v}^{2} \zeta_{v}
$$

Expression (5) contains designations for the average flow velocity $w_{v}$ for the working medium with density $\rho_{L}$ in the case of local resistance in the section $v=\overline{1, s}$ with a coefficient $\zeta_{v}$. 
The primary assessment of the Reynolds criterion when fulfilling (2) is carried out according to the empirical expression $\operatorname{Re}_{y}=353 Q_{1 \max } /\left(v_{1} D_{y}\right)$ [11]. This criterion connects the operating parameters of the throttling process: the nominal diameter $D_{y}$ from (2) and the maximum attainable flow rate $Q_{1 \max }, \mathrm{m}^{3} / \mathrm{h}$ through the valve for a working fluid with kinematic viscosity $v_{1}, \mathrm{~cm}^{2} / \mathrm{c}$ at temperature $t_{1},{ }^{\circ} \mathrm{C}$. In particular, we give set $U_{2}=\left\{U_{2 v}\right\}, v=\overline{1, n_{U_{2}}}$ as set for operating parameters and a complex $U_{3}=\left\{U_{3 v}\right\}, v=\overline{1, n_{U_{3}}}$ for the physic mechanical characteristics of the working medium where $\Delta P_{\min }$ is the minimum pressure drop; $\gamma_{0}$, $\mathrm{g} \times \mathrm{c} / \mathrm{cm}^{3}$ is the volumetric weight.

Taking into account the existing modes of fluid flow: laminar $(\operatorname{Re} \leq 10)$ and turbulent $\left(\operatorname{Re} \geq 10^{4}\right), \quad \mathrm{a}$ qualitative assessment of the coefficient of hydraulic resistance $\zeta_{12}$ for the transition region $\left(10<\operatorname{Re}<10^{4}\right)$ is made by summing the corresponding linear $\zeta_{1}$ and quadratic $\zeta_{2}$ components according to [10]

$$
\zeta_{12}=\zeta_{1}+\zeta_{2}
$$

Taking into account the Darcy-Weisbach formula for laminar flows [10-12] and the equation of fluid flow continuity, the following calculation of the specified linear component of the required hydraulic resistance coefficient $\zeta_{1}=\zeta_{1}\left(\psi_{\eta}\right)$ with pressure loss for total number of throttling passages $N=q_{1} q_{2}$ is proposed using dependence (3)

$$
\zeta_{1}\left(\psi_{\eta}\right)=\frac{4}{\operatorname{Re}_{y}} \frac{q_{1} q_{2}\left(\delta_{1}+\delta_{2}\right)}{\left[W\left(\psi_{\eta}\right)\right]^{2} H\left(\psi_{\eta}\right)}\left(\frac{D_{y} W_{m}}{d_{0}}\right)^{2}
$$

where is denoted: $W_{m} \equiv W\left[\psi_{\eta}(1 / 2)\right] / 2$.

The second component $\zeta_{2}=\zeta_{2}\left(\psi_{\eta}\right)$ from (6) for the quadratic part of the hydraulic resistance coefficient $\zeta_{12}$ corresponds to the turbulent flow of the working fluid and takes into account several stages of its formation. In particular, the following main stages are highlighted: (a) rotation by the bevel angle; (b) movement in channels of variable cross-section, annular and slotted. In case (b), the Borda formula [11] is applied in the approximation of equality of the areas of the narrowed and throttled channels. In addition, this takes into account the relationship between the inner and outer diameters of the separator from (1) with the nominal cross-sectional area of the wide channel during the annular and slotted movement of the working medium. Taking into account (1), (3), from (4) it follows

$$
\begin{gathered}
\zeta_{2}\left(\psi_{\eta}\right)=\frac{q_{1} \varphi}{90^{0}} \frac{D_{y}^{4} / 20}{\left[\left(D_{e x t 01}-2 \delta_{0}\right)^{2}-D_{e x t 02}{ }^{2}\right]^{2}}+ \\
+\frac{\sqrt{2}}{20} \frac{\left[\left(D_{e x t 01}-2 \delta_{0}\right)^{2}-D_{e x x 1}^{2}\right]^{2} D_{e x t 1}^{2}}{D_{y}^{6}} \times \\
\times\left[D_{i n t 1}\left(\lambda_{1}-L\right) /\left\{20 \pi q_{1} q_{2} W_{m} H\left(\psi_{\eta}\right) \varepsilon\left(\psi_{\eta}\right)\right\}-1\right]^{2}
\end{gathered}
$$

where the compression ratio of the jet $\varepsilon=\varepsilon\left(\psi_{\eta}\right)$ depending on the value of the central angle of the segment $\psi_{\eta}(\eta)$ for the open part of the throttle hole is calculated using the modified formula

$$
\varepsilon\left(\psi_{\eta}\right)=c_{0}+c_{1} /\left[c_{2}-n_{m}\left(\psi_{\eta}\right)\right]
$$

obtained on the basis of the Alshtul formula $[12,30]$. Here, the compression ratio of the jet in the modified form $n_{m}\left(\psi_{\eta}\right)=\left[n_{m 1}\left(\psi_{\eta}\right)+n_{m 2}\left(\psi_{\eta}\right)\right] / 2$ takes into account, respectively, the annular and slotted nature of the movement of the working medium according to (1), (3)

$$
\begin{gathered}
n_{m 1}\left(\psi_{\eta}\right) \equiv q_{1}\left(\delta_{1}+\delta_{2}\right)\left\{\lambda_{2}+d_{0}\left[1-z\left(\psi_{\eta}\right)\right]\right\} \times \\
\times\left(1+D_{i n t 1} / D_{\text {ext } 1}\right) /\left\{90^{0}\left[\left(D_{e x t 01}-2 \delta_{0}\right)^{2}-D_{i n t 1}^{2}\right]\right\}, \\
n_{m 2}\left(\psi_{\eta}\right) \equiv 8\left(L-\lambda_{1}\right) H\left(\psi_{\eta}\right) /\left(\pi d_{0}^{2}\right)
\end{gathered}
$$

Thus, summing the obtained components from (7), (8) for the desired coefficient of hydraulic resistance in accordance with expression (6), we have

$$
\begin{aligned}
& \zeta_{12}\left(\psi_{\eta}\right)=g_{1} /\left\{H\left(\psi_{\eta}\right)\left[W\left(\psi_{\eta}\right)\right]^{2}\right\}+ \\
& +g_{2}+g_{4}\left\{g_{3} /\left[H\left(\psi_{\eta}\right) \varepsilon\left(\psi_{\eta}\right)\right]-1\right\}^{2}
\end{aligned}
$$

where given (9) - (11) accepted

$$
\begin{gathered}
\varepsilon\left(\psi_{\eta}\right)=c_{0}+c_{1} /\left\{c_{2}-\left[g_{6}+g_{51}-g_{52} \eta\left(\psi_{\eta}\right)\right] / 2\right\}, \\
\eta\left(\psi_{\eta}\right)=\left(\psi_{\eta}-\sin \psi_{\eta}\right) / \pi, g_{51}=q_{1}\left(\lambda_{2}+d_{0}\right), \\
g_{1} \equiv 4 q_{1} q_{2}\left(\delta_{1}+\delta_{2}\right)\left(D_{y} W_{m}\right)^{2} /\left(\operatorname{Re}_{y} d_{0}^{2}\right), \\
g_{2} \equiv q_{1} \varphi\left(D_{y}^{4} / 20\right)\left\{90^{0}\left[\left(D_{e x t 01}-2 \delta_{0}\right)^{2}-D_{e x t 02}{ }^{2}\right]^{2}\right\}, \\
g_{3} \equiv D_{i n t 1}\left(\lambda_{1}-L\right) /\left(20 \pi q_{1} q_{2} W_{m}\right), \\
g_{4} \equiv \sqrt{2}\left[\left(D_{e x t 01}-2 \delta_{0}\right)^{2}-D_{e x t 1}{ }^{2}\right]^{2} D_{e x t 1}{ }^{2} /\left(20 D_{y}^{6}\right), \\
g_{50} \equiv q_{1}\left(\delta_{1}+\delta_{2}\right)\left\{\lambda_{2}+d_{0}\left[1-z\left(\psi_{\eta}\right)\right]\right\} \times \\
\times\left(1+D_{i n t 1} / D_{e x t 1}\right) /\left\{90^{0}\left[\left(D_{e x t 01}-2 \delta_{0}\right)^{2}-D_{i n t 1}{ }^{2}\right]\right\}, \\
g_{51} \equiv q_{1}\left(\lambda_{2}+d_{0}\right), g_{52} \equiv g_{50} d_{0} .
\end{gathered}
$$

The proposed analytical method for calculating the coefficient of hydraulic resistance $\zeta_{12}\left(\psi_{\eta}\right)$ using (12) makes it possible to analyze the dependence of the conditional throughput of the valve $K_{v y}\left(\psi_{\eta}\right)$ on the 
central angle of the segment $\psi_{\eta}(\eta)$ for the open part of the throttle hole using the known relationship [10-12]

$$
K_{V y}\left(\psi_{\eta}\right)=5.04 \cdot 10^{4} \pi D_{y}^{2}\left[\zeta_{12}\left(\psi_{\eta}\right)\right]^{-1 / 2}
$$

Therefore, using (13), it is also possible to estimate the throughput characteristic of pipeline fittings of this class and solve the corresponding problem of profiling.

\section{Results and Discussion}

We will present an illustration of the results of this study using the example of a qualitative assessment of the hydraulic resistance coefficient $\zeta_{12}$ for the transitional region of the flow of the working medium ( $10<\operatorname{Re}<10^{4}$ ) in the flowing part of a direct-flow valve with an external butterfly valve [16].

According to the proposed expression (12) for $\zeta_{12}\left(\psi_{\eta}\right)$, the character of the functional dependence of the sought coefficient on the design parameters of the liquid throttling process from the set (1) for $U_{1}=\left\{U_{1 v}\right\}, v=\overline{1, n_{U_{1}}}$ is studied for within the selected limits of their change.

In accordance with the accepted requirements for industrial testing of control valves, it is considered that the working fluid is water with the following physical and mechanical characteristics $U_{3}=\left\{U_{3 v}\right\}, v=\overline{1, n_{U_{3}}}$ : density $\rho_{L}=10^{3} \quad \mathrm{~kg} / \mathrm{m}^{3} ; \quad$ kinematic viscosity $v_{1}=0.81 \times 10^{-2} \mathrm{~cm}^{2} / \mathrm{c}$ and bulk density $\gamma_{0}=0.995$ $\mathrm{g} \times \mathrm{c} / \mathrm{cm}^{3}$ at temperature $t_{1}=30{ }^{\circ} \mathrm{C}$.

The values of the operating parameters $U_{2}=\left\{U_{2 v}\right\}, v=\overline{1, n_{U_{2}}}$ for the process of throttling of the working fluid are different: the maximum attainable flow rate through the valve $Q_{1 \max }=0.8 \mathrm{~m}^{3} / \mathrm{h} ;$ minimum pressure $\Delta P_{\text {min }}=1.3 \mathrm{kPa}$; temperature $t_{1}=30{ }^{\circ} \mathrm{C}$.

From a set of design parameters, we indicate values for (1): $D_{\text {ext } 1}=3.68 \times 10^{-2} \mathrm{~m} ; \quad D_{\text {int } 1}=3.4 \times 10^{-2} \mathrm{~m}$; $D_{\text {ext } 01}=6.5 \times 10^{-2} \quad \mathrm{~m} ; \quad D_{\text {ext } 02}=5.3 \times 10^{-2} \quad \mathrm{~m}$; $L=2.35 \times 10^{-2} \mathrm{~m} ; d_{0}=2.0 \times 10^{-3} \mathrm{~m} ; \lambda_{1}=1.0 \times 10^{-3} \mathrm{~m}$; $\lambda_{2}=3.9 \times 10^{-4} \mathrm{~m} ; \delta_{1}=\delta_{2}=2.8 \times 10^{-3} \mathrm{~m} ; \delta_{0}=1.2 \times 10^{-2}$ $\mathrm{m} ; q_{1}=24 ; q_{2}=7 ; \varphi=45^{\circ}$.

The obtained values for the central angle of the segment $\psi_{\eta}$ for the open part of the throttle hole, the data for the nominal diameter $D_{y}$ and the Reynolds criterion $\operatorname{Re}_{y}$ depending on the degree of valve opening $\eta$ are given in Table 1.

As can be seen from Table 2, in the indicated ranges of change in the values of the separator parameters, an increase in the degree of valve opening from $\eta_{1}=0.2$ to $\eta_{2}=0.5$ leads to a decrease in the value $\zeta_{12}$ by 19.6 times, and when changing from $\eta_{2}=0.5$ to $\eta_{4}=1.0$, a decrease in the studied characteristic by 42.4 times is observed. At the same time, an increase in the degree of valve opening from $50 \%$ to $100 \%$ provides an increase in the value of its conditional throughput $K_{V y}$ by 2.9 times. These facts prove the effectiveness of the proposed method for throttling the flows of the working medium in the main unit of the control valve "separatorrotary lock" [16].

Table 1. Results of calculating for the central angle of the segment $\psi_{\eta}$ for the open part of the throttle hole, the data for the nominal diameter $D_{y}$ and the Reynolds criterion $\operatorname{Re}_{y}$ depending on the degree of valve opening $\eta$.

\begin{tabular}{|c|c|c|c|c|}
\hline$v$ & $\eta_{v}$ & $\psi_{\eta v}, \operatorname{rad}$ & $D_{y v}, 10^{-2}, \mathrm{~m}$ & $\operatorname{Re}_{y v}, 10^{4}$ \\
\hline 1 & 0.2 & 1.627 & 1.159 & 6.6754 \\
\hline 2 & 0.5 & 2.309 & 1.833 & 4.2219 \\
\hline 3 & 0.8 & 2.824 & 2.319 & 3.3377 \\
\hline 4 & 1.0 & 3.142 & 2.592 & 2.9853 \\
\hline
\end{tabular}

The calculated values of the hydraulic resistance coefficient $\zeta_{12}$ for the transitional region of the flow of the working fluid and the conditional throughput of the valve $K_{V y}$ according to expressions (12), (13) are contained in Table 2.

Table 2. Results of calculating of the hydraulic resistance coefficient $\zeta_{12}$ for the transitional region of the flow of the working fluid and the conditional throughput of the valve $K_{V y v}$ depending on the degree of valve opening $\eta$

\begin{tabular}{|c|c|c|c|}
\hline$v$ & $\eta_{v}$ & $\zeta_{12 v}, 10^{1}$ & $K_{V y v}, \mathrm{~m}^{3} / \mathrm{h}$ \\
\hline 1 & 0.2 & 61.832 & 0.214 \\
\hline 2 & 0.5 & 3.159 & 2.367 \\
\hline 3 & 0.8 & 1.532 & 5.437 \\
\hline 4 & 1.0 & 1.459 & 6.964 \\
\hline
\end{tabular}

According to the data obtained [13], to estimate the specified coefficient in the case of throttling of the fluid flow in an axial valve with a movable external lock [25], a number of the most significant design parameters have been identified, which makes it possible to carry out similar reasoning in this work. The calculations performed for the dependence $\zeta_{12}\left(\psi_{\eta}\right)$ allowed us to analyze the influence of various design parameters on the process under study in the flow part of a direct-flow valve with an external rotary valve [16]. The group of surfaces for the functions $\zeta_{12}\left(d_{0}, q_{2}\right), \zeta_{12}\left(d_{0}, \delta_{1}\right)$, $\zeta_{12}\left(D_{\text {int } 1}, q_{1}\right)$ obtained using expression (12) are shown, respectively, in Fig. 1a, 1b; Fig. 2a, 2b; Fig. 3a, 3 b.

Moreover, the graphs in Fig. 1a-3a are plotted for the value of the valve opening degree $\eta_{1}=0.2$, and on the others Fig. $1 b-3 b$ are for values from a range $\eta \in[0.5 ; 1.0]$. There is a smooth drop in the hydraulic resistance coefficient $\zeta_{12}$ with an increase in the values of the diameter $d_{0}$ of the throttling holes (graphs 1-3, Fig. 1b; graphs 1-3, Fig. 2b), as well as their number $q_{1}$ in one row on the cylindrical surfaces of the separator 
and the outer rotary valve shell (Fig. . 3a; graphs 1-3, Fig. 3b).

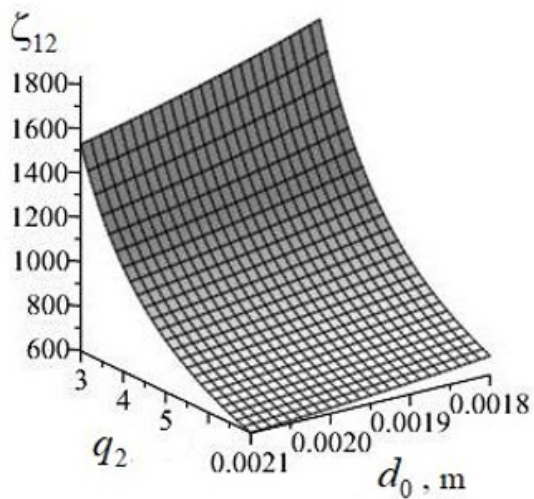

a)

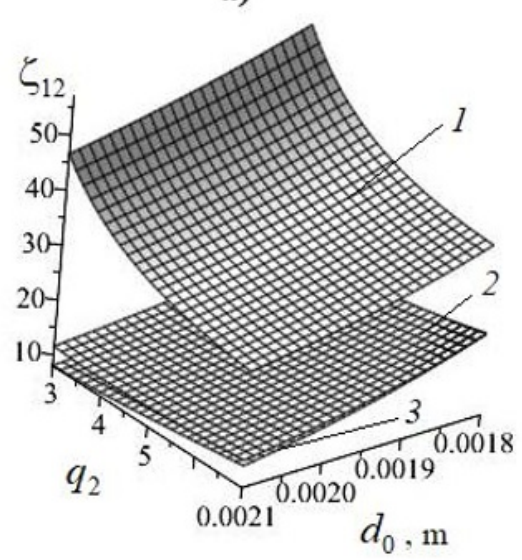

b)

Fig. 1. Relationship between the coefficient of hydraulic resistance $\zeta_{12}$, the diameter of the throttling holes $d_{0}$ and the number of their rows $q_{2}: q_{1}=24 ; D_{\text {int } 1}=3.4 \times 10^{-2} \mathrm{~m}$; $\delta_{1}=\delta_{2}=2.8 \times 10^{-3} \mathrm{~m}$; a) $\eta=0.2$; b) $1-\eta=0.5 ; 2-$ $\eta=0.8 ; 3-\eta=1.0$.

The indicated tendency is more pronounced at small degrees of valve opening - near the value $\eta_{1}=0.2$ (Fig. 1a; Fig. 2a; Fig. 3a). In particular, with the following fixed values of design parameters $q_{1}=24 ; q_{2}=7$; $D_{\text {int } 1}=3.4 \times 10^{-2} \mathrm{~m} ; \delta_{1}=\delta_{2}=2.8 \times 10^{-3} \mathrm{~m}$ an increase in the diameter of the throttling holes within $d_{0}=(1.8-2.1) \times 10^{-3} \mathrm{~m}$ leads to a drop in the value of the hydraulic resistance coefficient by 1.12 times. Significant upward changes in coefficient values $\zeta_{12}$ are associated with a reduction in the number of rows of throttling holes $q_{2}$ (Fig. 1a; Fig. 1b).

In addition, there is a tendency to a decrease in the value of the hydraulic resistance coefficient with a decrease in parameters $\delta_{1}$ (Fig. 2a; Fig. 2b) and (Fig. 3a; Fig. 3b). These facts create conditions for finding rational ranges of changes in design parameters.

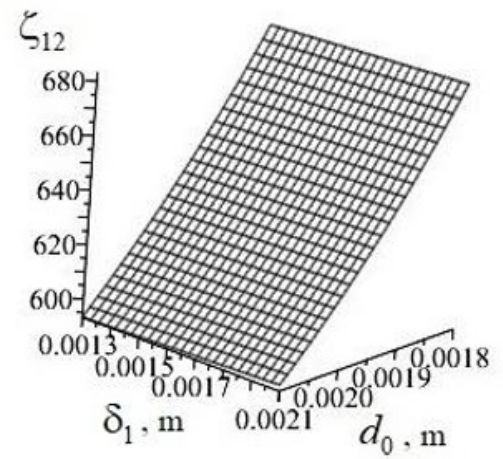

a)

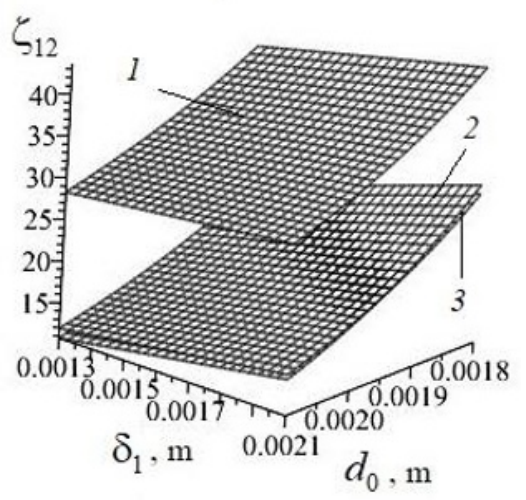

b)

Fig. 2. Relationship between the coefficient of hydraulic resistance $\zeta_{12}$, the diameter of the throttling holes $d_{0}$ and the wall thickness of the separator $\delta_{1}: q_{1}=24 ; q_{2}=7$; $D_{\text {int } 1}=3.4 \times 10^{-2} \mathrm{~m}$; a) $\eta=0.2$; b) $1-\eta=0.5 ; 2-$ $\eta=0.8 ; 3-\eta=1.0$

The influence of the function $\zeta_{12}\left(\psi_{\eta}\right)$ obtained based on the proposed expression (12) on the parameters of the conditional throughput $K_{V y}$ are presented in Fig. 4. In particular, the calculation $\zeta_{12}\left(\psi_{\eta}\right)$ according to (12) made it possible, when using (13), to trace the tendency of an increase in the throughput characteristic with an increase in the valve opening degree (see Table 2, Fig. 4).

In addition, in Fig. 4 shows a clear increase in the throughput of the separator with an increase in the diameter of the throttling holes, and more significant at values of the valve opening degree $\eta$, in the vicinity of unity (graph 4, Fig. 4).

Thus, the estimation of the nominal throughput $K_{V y}$ depending on the design parameters of the direct-flow valve can be used in the analysis of its throughput characteristics and the choice of profiling for the efficient operation of control valves. 


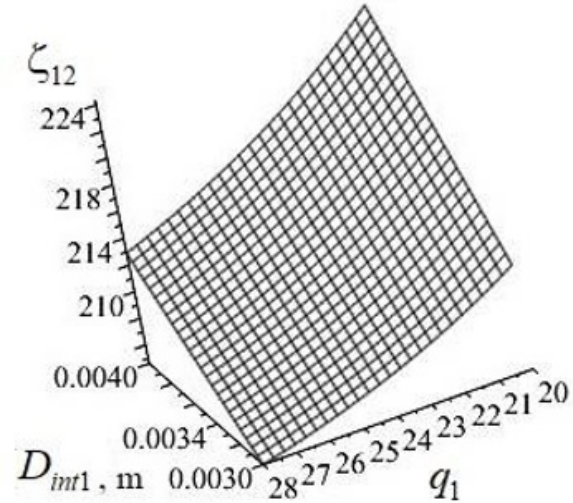

a)

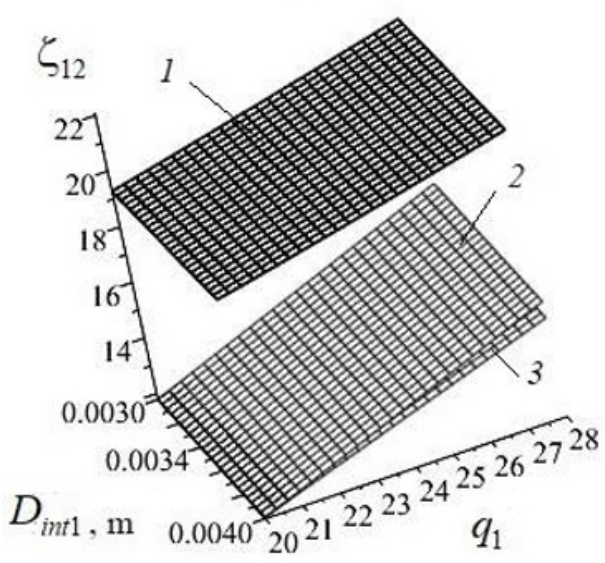

b)

Fig. 3. Relationship between the coefficient of hydraulic resistance $\zeta_{12}$, the inner diameter $D_{i n t 1}$ of the cage and the number of throttling holes in one row $q_{1}: q_{2}=7$;

$d_{0}=2.0 \times 10^{-3} \mathrm{~m} ; \delta_{1}=\delta_{2}=2.8 \times 10^{-3} \mathrm{~m} ;$ a) $\eta=0.2 ;$ b) $1-$ $\eta=0.5 ; 2-\eta=0.8 ; 3-\eta=1.0$

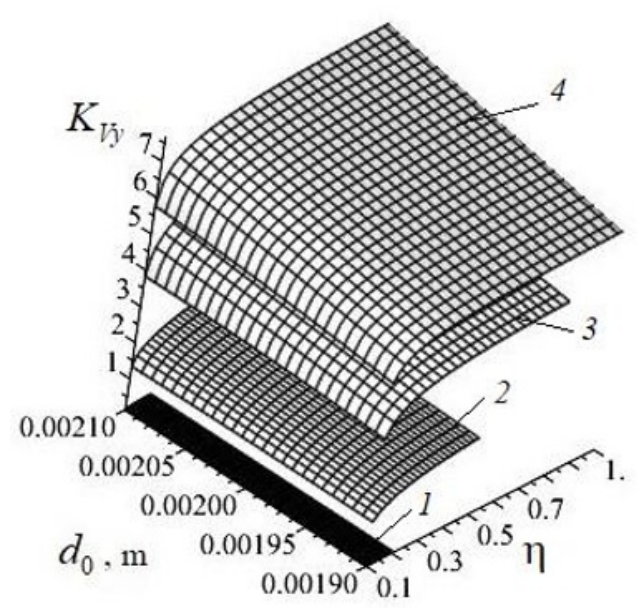

Fig. 4. Relationship between the conditional throughput of the valve $K_{V y v}$, the degree of valve opening $\eta$ and the diameter of the throttling holes $d_{0}: q_{1}=24 ; q_{2}=7 ; D_{\text {int } 1}=3.4 \times 10^{-2} \mathrm{~m}$; $\delta_{1}=\delta_{2}=2.8 \times 10^{-3} \mathrm{~m} ; 1-\eta=0.2 ; 2-\eta=0.5 ; 3-$ $\eta=0.8 ; 4-\eta=1.0$

\section{Conclusions}

In the present work, a qualitative assessment of the hydraulic resistance coefficient is carried out when the throttling of fluid flows in the "separator-butterfly valve" unit [16] is carried out, depending on the design and operating parameters of the process by an analytical method. The results obtained are an integral part of the theoretical basis for the design of pipeline valves and contribute to the modeling of the throttling process, including on the basis of a stochastic approach [31], and the choice of rational ranges of change in the design parameters of the process under study.

The main results include the theoretical confirmation of the effectiveness of the proposed method for throttling the flows of the working medium in the main unit of the control valve "separator - external rotary lock". In particular, it was found that, within the selected range of variation of the separator parameters, an increase in the valve opening degree from $20 \%$ to $50 \%$ leads to a decrease in the hydraulic resistance coefficient by 19.6 times, and an increase in this degree from $20 \%$ to $100 \%$ is associated with a decrease in the studied characteristic 42.4 times. In addition, conditions have been created for assessing the throughput characteristics of pipeline valves of the specified class and solving the corresponding problem of profiling.

The results obtained are used to study the influence of the main design parameters of the "separator-rotary lock" unit on the flow capacity of the control valve and are relevant for stochastic modeling of the hydrodynamic cavitation process.

So, the conclusions and results of the work include the following:

- An analytical method is proposed for calculating the hydraulic resistance coefficient in the flow path of a control valve with a rotary gate, taking into account the sets of design and operating parameters, as well as the physical and mechanical characteristics of the working medium.

- The efficiency of the proposed method of throttling the flows of the working medium in the main unit of the control valve "separator - external rotary gate" [16] is shown on the basis of the calculation of the hydraulic resistance coefficient.

- A set of the most significant design parameters of the liquid flow throttling process for the specified control valve assembly was identified when analyzing the corresponding dependencies for the hydraulic resistance coefficient.

- Preconditions have been created for calculating the flow characteristic of a direct-flow control valve of the specified class and solving the problem of profiling.

- An integral part of the theoretical basis for modeling the process of liquid throttling in the "separator - external butterfly valve" control valve unit, including the stochastic one, has been formed. 


\section{References}

[1] Flowserve, Linear control valves [Electronic resource] Available at: https://www.flowserve.com/en/products/valves/line ar-control-valves (accessed: 27 October 2020)

[2] Mokveld, Axial flow valves by Mokveld [Electronic resource] (2020) Available at: https://mokveld.com/en/home (accessed: 27 October 2020)

[3] R.T. Knapp, J.W. Daily, F.G. Hammitt, Cavitation (New York, McGraw-Hill, 1970) 578

[4] S. Xu, Y. Qiao, X. Liu, C. Church, M. Wan, Fundamentals of Cavitation, In: M. Wan, Y. Feng, G. Haar (eds.), Cavitation in Biomedicine (Springer, Dordrecht, 2015) DOI: 10.1007/978-94017-7255-6_1

[5] J.-P. Franc, J.-M. Michel, Fluid Mechanics and Its Applications 76, 11, 2005 DOI: 10.1007/1-40202233-6

[6] T.J. Preston, Valve, KR20180055897A, F16K 3/26, F16K 3/26, F16K 3/314 (25 May 2018)

[7] V. Esveldt, Throttle valve, Patent US8297315B2, F16K 47/08 (30 October 2012)

[8] H.H. Weevers, Control valve, Patent US4327757, F16K 47/14 (4 May 1982)

[9] F. Ziegler, Piston valve controlled by own medium [Eigenmedium gesteuertes Ringkolbenventil], Patent DE102013108940B4 Germany, F16K 1/126 (5 December 2019)

[10] E.S. Arzumanov, Gidravlicheskiye reguliruyushchiye organy sistem avtomatizirovannogo upravleniya [Hydraulic regulatory bodies of automated control systems]. (Moscow, Mashinostroyeniye [Engineering], 1985) 256

[11] E.S. Arzumanov, Raschet $i$ vybor reguliruyushchikh organov avtomaticheskikh system [Calculation and selection of the regulatory bodies of automatic systems] (Moscow, Energy, 1971) 112

[12] A.D. Altshul, Gidravlicheskoye soprotivleniye [Hydraulic resistance] (Moscow, Nedra, 1982) 224

[13] A. Kapranova, S. Neklyudov, A. Lebedev, A. Meltser, Int. J. Mech. Eng. Technol. 9, 8, 153159 (2018)

[14] A.B. Kapranova, A.E. Lebedev, S.V. Neklyudov, A.M. Melzer, Frontiers in Energy Research: Process and Energy Systems 8, 32, 1-17 (March 2020) DOI: $10.3389 /$ fenrg.2020.00032

[15] A.B. Kapranova, A.E. Lebedev, A.M. Melzer, S.V. Neklyudov, About Formation of Elements of a Cyber-Physical System for Efficient Throttling of Fluid in an Axial Valve. In: A. Kravets, A. Bolshakov, M. Shcherbakov (eds), CyberPhysical Systems: Advances in De-sign \& Modelling. Studies in Systems, Decision and Con- trol 259, 109-119 (Springer, Cham, 2020) DOI: 10.1007/978-3-030-32579-4_9

[16] A.E. Lebedev, A.B. Kapranova, A.M. Melzer, S.A. Solopov, D.V. Voronin, V.S. Neklyudov, E.M. Serov, Utility Patent 2657371 Russian Federation, Direct-flow control valve, IPC F16K 1/12 17 (Publ. 06.13.2018)

[17] G. Volgin, E3S Web of Conferences 97, 05031 (2019) DOI: 10.1051/e3sconf/20199705031

[18] V.N. Kireev, A. Nizamova, S.F. Urmancheev, The hydraulic resistance of thermoviscous liquid flow in a plane channel with a variable cross-section, Journal of Physics Conference Series 1158, 3, $032014 \quad$ (2019) DOI: 10.1088/1742$6596 / 1158 / 3 / 032014$

[19] V.D. Narasimhamurthy, H. Andersson, International Journal of Heat and Fluid Flow, 51 (2014) DOI: 10.1016/j.ijheatfluidflow.2014.10.008

[20] V.D. Narasimhamurthy, H. Andersson, DNS of turbulent flow in a rotating rough channel, Conference: 8th Workshop on Direct and LargeEddy Simulation 15 (January 2011) DOI: 10.1007/978-94-007-2482-2_65

[21] G. Alfonsi, Applied Mechanics Reviews 64, 2, 0802 (2011) DOI: 10.1115/1.4005282

[22] A.B. Kapranova, A.E. Lebedev, S.A. Solopov, A.M. Melzer, Czasopismo techniczne, Mechanika 113, 2, 136-144 (2016)

[23] A. Kapranova, S. Neklyudov, A. Lebedev, A. Melzer, Int. J. Mech. Eng. Technol. 9, 8, 160-166 (2018)

[24] A. Kapranova, A. Lebedev, A. Melzer, S. Neklyudov, Int. J. Mech. Eng. Technol. 9, 3, 2531 (2018)

[25] A.E. Lebedev, A.B. Kapranova, A.M. Melzer, S.A. Solopov, D.V. Voronin, V.S. Neklyudov, Direct-acting control valve, Patent on the model 175776 Russian Federation, IPC F16K 1/12, F16K 47/14, F16K 3/24 (May 2018)

[26] A. Kapranova, A. Lebedev, A. Melzer, S. Neklyudov, E3S Web of Conference 140, 06005, (2019) DOI: 10.1051/e3sconf/201914006005

[27] E.F. Sheaffer, Design of piping systems, Pullman power products a wiley-interscience publication (New York, Chichester, Brisbane, Toronto, A wiley interscience publication john wiley sons, 1977) 436

[28] M. Frankel, Facility piping systems handbook, 2nd ed. (New York, Chicago, San Francisco, Lisbon, London, Madrid, Mexico, City Milan, New Delhi, San Juan, Seoul, McGraw-Hill, 2002) 324

[29] C. Canali, Standards for design of piping support (Snamprogetti, 2001) 226

[30] I.E. Idelchik, Spravochnik po gidravlicheskim soprotivleniyam [Hydraulic resistance reference book] (Moscow, Mashinostroyeniye [Mechanical Engineering], 1975) 559 
[31] A.B. Kapranova, A.E. Lebedev, S.V. Neklyudov, A.M. Melzer, Vestnik IGEU 3, 58-64 (2020) DOI: 10.17588/2072-2672.2020.3.058-064 